IEEE MICROWAVE AND WIRELESS COMPONENTS LETTERS

\title{
A Wide-Band, 5-Tap Transversal Filter With Improved Testability for Equalization up to $84 \mathrm{~Gb} / \mathrm{s}$
}

Yu Ban, Timothy De Keulenaer, Zhisheng Li, Joris Van Kerrebrouck, Student Member, IEEE, Jeffrey H. Sinsky, Senior Member, IEEE, Bartek Kozicki, Johan Bauwelinck, Member, IEEE, and Guy Torfs, Member, IEEE

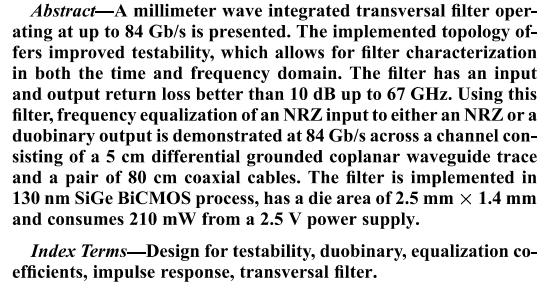
Index Terms-Design for testability, duobinary,
efficients, impulse response, transieral fiter

I. INTRODUCTION

$\mathbf{T}^{\mathrm{H}}$

HE data rate of chip-to-chip/chip-to-module interconnects continuously increases to meet the demand for greater network capacity. However, operating at high data rates poses great challenges as it requires a very high bandwidth (BW) component across the electrical link, while increased data rates face excessive frequency dependent attenuation, reflection and inter-symbol interference (ISI) [1]. As a result, in high-speed communication links, the received data waveforms can be significantly distorted after travelling through coaxial cables or printed circuit board (PCB) interconnects [2]. To compensate signal degradation and to enhance the channel capacity, advanced modulation and/or equalization are widely used in transmitter and receiver circuits. Towards that end, duobinary modulation format holds great potentia that end, dubing for attaining high-specd or long reach electical transmission, as it utilizes the avallable BW more eficiently compared to non-return-to-zero (NRZ) and allows for the confinement of a signal's power spectral density (PSD) to lower frequencies [3]. A duobinary signal is defined as the sum of the present bit an

Manuscript received May 11, 2015; revised July 29, 2015; accepted Augu 12, 2015. This work was supported by the Agency for Innovation by Science
and Technology in Flanders (IWT) and the Special Research Fund (BOF) of Ghent University Y. Ban, T. De Keulenaer, Z. Li, J. Van Kerrebrouck, J. Bauwelinck and G.

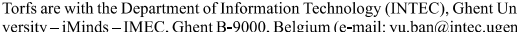

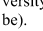

J. H. Sinsky is with Bell Labs, Alcatel-Lucent, Holmdel, NJ 07733 USA. B. Kozicki is with Bell Labs, Alcatel-Lucent, Antwerp 2018, Belgium. Color versions of

Digital Object Identifier 10.1109/LMWC.2015.2479844 the previous bit in a binary sequence, which is expressed in the frequency domain in (1), where $T_{s}$ represents the bit period

$$
H(f)=\frac{Y(f)}{X(f)}=\frac{1}{2} \cdot\left[1+\exp \left(-j 2 \pi f T_{s}\right)\right] .
$$

The original data can easily be recovered using a XOR gate at the receiver [2]

In this Letter, a 5-tap feed-forward equalizer (FFE) transversal filter extended with test buffers is presented. This proposed topology introduces an extra signal path along the transversal filter, such that the FFE can be individually characterized, in both frequency- and time-domain. Optimizing FFE tap weights with a least square error (LSE) fitting, the transmission channel is equalized for two data formats, either standard two-level NRZ signaling or three-level duobinary signaling A serial electical transion, for both modulation signaling. A serial electrical transmission, for both modulation schemes is demonstrated at $84 \mathrm{~Gb}$.

II. Proposed FFE Topology

Using conventional FFE topologies [4], [5], a problem occurs when the FFE is integrated in a transmitter and needs to be haracterized. Adding test ports next to the conventional input and output ports is a solution, however, it requires extra circuits such as switches in the high speed data path, which will increase the power consumption and degrade the signal quality due to dition cence ementer using a transmission line (TL), which requires an on-chip termination. In this Letter, by replacing this termination with additional test buffers, an extra path is introduced, so that the FFE can be used both in clockwise and counter-clockwise directions. During normal operation, these buffers are disabled, but their output loads will still function as TL terminations. The proposed topology is shown in Fig. 1

The input test buffer and output test buffer are directly connected to chip IO pads, allowing us to probe the equizer separately. Due to the symmetrical ature of be supplied on both left- and right-hand side (shown in Fig. 1). At the left-hand side of the FFE, the input buffer connects with the output of an on-chip multiplexer (MUX) which combines four lower rate streams into an $84 \mathrm{~Gb} / \mathrm{s}$ differential NRZ signal. The linear output buffer drives the equalized signal over an external channel. At the right-hand side of the FFE, applying an additional test input and output buffer, the FFE functionality can be also measured, either in frequency- or time-domain. However, the FFE is driven in the opposite direction. Therefore, the 

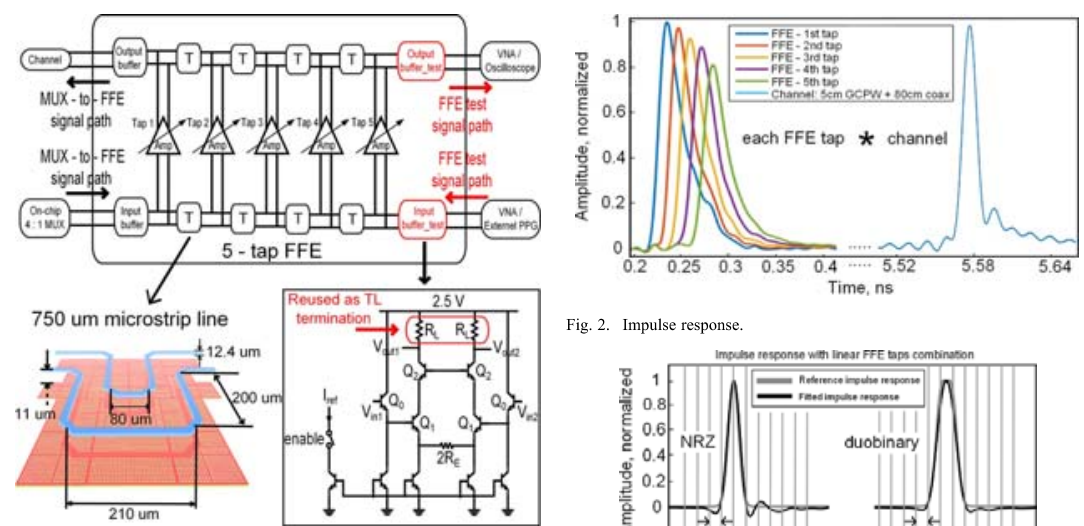

Fig. 2. Impulse respons.

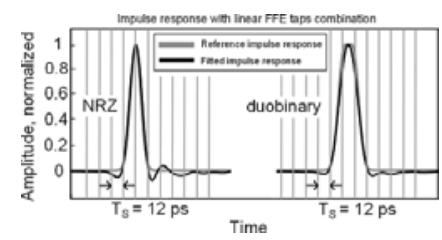

Fig. 1. Fully integrated FFE structure.

FFE tap weights have to be mirrored when employing this data path. Each input and output buffer has an individual enable/disable switch, in order to choose the operating mode.

In comparison to the conventional FFE topology with on-chip resistors as delay line terminations [5], [6], the introduced signal path ("FFE test signal path" as shown in Fig. 1) allows us to characterize the FFE individually without disconnecting the MUX And the measured response of $a$ FFE tap helps to do 政 the difficult 5-dimension FFE parameter to obtain optimal channel equalization.

The relationship of the FFE input and output is given in (2). $A_{i}$ represents the tap weight and $T a p_{\text {delay }}$ represents the time delay between neighboring taps

$$
Y(t)=\sum_{i=1}^{5}\left(A_{i} \cdot X\left(t-(i-1) \cdot \operatorname{Tap}_{\text {delay }}\right\}\right) .
$$

this linear combination are optimized using a gradient search algorithm to obtain a LSE fit between the simulated response and a desired reference pulse [8]. In this way, the optimal value of pre-cursors and post-cursors in the FFE is selected. The resulting impulse response after optimization for a serial data rate of $84 \mathrm{~Gb} / \mathrm{s}$ is depicted in Fig. 3 .

\section{IMPULSE RESPONSE}

By using high frequency probes with a pitch of $150 \mu \mathrm{m}$, the frequency response of the integrated FFE is measured. Based on the response of each FFE tap at the maximum gain, the impulse response of each FFE tap is calculated and presented in Fig. 2. It can be observed that each FFE tap is separated in time by a delay of $12.4 \mathrm{ps}$, corresponding to the delay introduced by the on-chip
TL. The later taps have a lower output power, which is caused TL. The later taps have a lower output power, which is caused by the frequency-dependent loss introduced by the on-chip TLs. in the experiments, consisting of a $5 \mathrm{~cm} \mathrm{RO} 4003 \mathrm{C}$ differential grounded coplanar waveguide (GCPW) trace [7] and a pair of $80 \mathrm{~cm}$ coaxial cables.

The equalized channel response can be simulated by convolving the measured channel response with a linear combination of the measured impulse responses. The coefficients of

Fig. 3. Equalized channel response fitting to NRZ or duobinary.

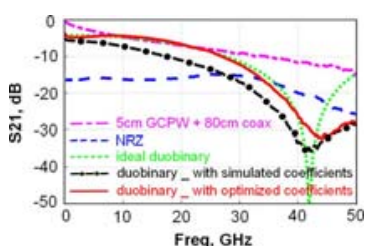

IV. FREQUENCY- AND TIME-DOMAIN VERIFICATION

The FFE is evaluated in the frequency-domain using an Agilent PNA-X N5247A. The methodology proposed above provides a good starting point for the FFE coefficients. As the positive and negative coefficients of the FFE tap are assumed to be exactly balanced in the simulation, during measurements, further optimization is performed by looking at the FFE frequency response on the VNA. Fig. 4 shows the measured uncompensated channel as well as the channel optimized for NRZ, the reference response of a duobinary channel, the measured response using the FFE pared response using the FFE parameters obtained with the above deThe optinized dubinary reponse closely mponse. The optimized duobinary reponse closely matches the ref-
erence response. The NRZ reponse is BW limited to $40 \mathrm{GHz}$, 


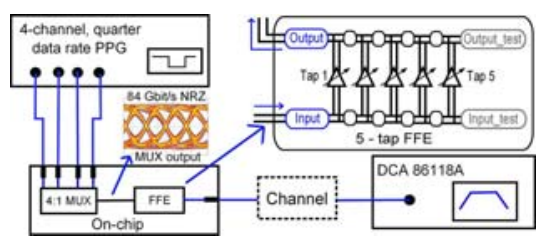

Fig. 5. Time-domain measurement setup.

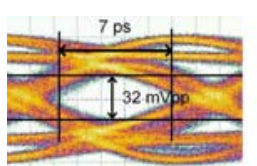

$15 \mathrm{mV}, 1.5 \mathrm{ps} / \mathrm{div}$

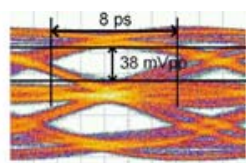

$25 \mathrm{mV}, 1.5 \mathrm{ps} / \mathrm{div}$
Fig. 6. Equalized $84 \mathrm{~Gb} / \mathrm{s}$ output. (a) NRZ. (b) duobinary.

4:1 Mux

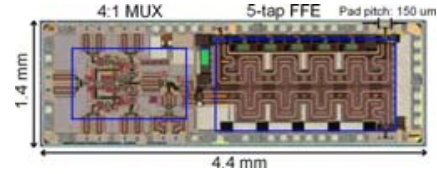

Fig. 7. Die micrograph.

TABLE I

TABLE I
Performance Comparison With Previous Publications

\begin{tabular}{|c|c|c|c|c|}
\hline Ref. & [4] & [5] & [9] & This work \\
\hline Process & $65 \mathrm{~nm} \mathrm{CMOS}$ & $180 \mathrm{~nm} \mathrm{SiGe}$ & $130 \mathrm{~mm}$ SiGe & $130 \mathrm{~nm}$ SiGe \\
\hline $\begin{array}{l}\text { Topology } \\
\text { Data rate }\end{array}$ & $\begin{array}{l}\text { 4-tap FFE } \\
64 \mathrm{~Gb} / \mathrm{s}\end{array}$ & $\begin{array}{c}\text { 7-tap FFE } \\
49 \mathrm{~Gb} / \mathrm{s}\end{array}$ & $\begin{array}{c}\text { 1-tap DFE } \\
80 \mathrm{~Gb} / \mathrm{s}\end{array}$ & $\begin{array}{l}\text { 5-tap FFE } \\
84 \mathrm{~Gb} / \mathrm{s}\end{array}$ \\
\hline Output swing & $600-850 \mathrm{mV}_{\mathrm{PP}}$ & $4900 / 3$ & socors & $500 \mathrm{mV}_{\mathrm{PP}}$ \\
\hline Power & $78 \mathrm{~mW}$ & $750 \mathrm{~mW}$ & $4000 \mathrm{~mW}$ & $210 \mathrm{~mW}^{*}$ \\
\hline FoM ${ }^{* *}$ & $1.2 \mathrm{p} / / \mathrm{bit}$ & $15.3 \mathrm{p} / / \mathrm{bit}$ & $50 \mathrm{pJ} / \mathrm{bit}$ & $2.5 \mathrm{pJ} / / \mathrm{bit}$ \\
\hline
\end{tabular}

"Total chip power, including MUX, is $750 \mathrm{~mW}$
${ }^{*}$ FoM $=$ Power / Data Rate.

which will degrade the eye opening as will be seen during the time-domain measurements.

The FFE functionality is verified in the time-domain using an Agilent $86100 \mathrm{C}$ mainframe and $70 \mathrm{GHz} 86118 \mathrm{~A}$ remote sampling heads. As discussed in Section II, thanks to the test configuration, the proposed FFE can be measured in two directions, with the FFE input either from the on-chip MUX, or from an external pulse pattern generator (PPG). As the maximum bit rate of our available PPG is limited to $50 \mathrm{~Gb} / \mathrm{s}$, the on-chip MUX and the counter-clockwise path of the FFE is enabled to perform the transient measurements. The experimental setup using the MUX-to-FFE signal path is depicted in Fig. 5. The FFE output MUX-to-FFE signal path is depicted in Fig. 5 . The FFE output
port is connected to the sampling oscilloscope, via a $5 \mathrm{~cm}$ differential GCPW trace and a pair of $80 \mathrm{~cm}$ coaxial cables, to evaluate the eye diagram for signal equalized with the proposed FFE and transmitted over the channel for both NRZ and duobinary signaling at $84 \mathrm{~Gb} / \mathrm{s}$.

By mirroring the FFE tap weights, the eye diagrams of the equalized NRZ and duobinary output at $84 \mathrm{~Gb} / \mathrm{s}$ are captured after the channel, as presented in Fig. 6 . The NRZ eye opening shows some BW limitation as expected from the frequency-domain measurement. On the other hand, the duobinary eye is constructed at the output of the channel, with a larger eye opening (vertical: $38 \mathrm{mV}$ vs. $32 \mathrm{mV}$, horizontal: 8 ps vs. $7 \mathrm{ps}$ ). Without equalization, the eye is completely closed.

The total chip, including a FFE and a MUX, is fabricated in $130 \mathrm{~nm}$ STMicroelectronics SiGe BiCMOS technology, featuring a $f_{T}$ value of over $200 \mathrm{GHz}$. Table I illustrates the performance comparison with previously published equalizers beyond $40 \mathrm{~Gb} / \mathrm{s}$ [9]. Fig. 7 presents die micrograph of the chip.

\section{CONCLUSION}

In this Letter, we have demonstrated an $84 \mathrm{~Gb} / \mathrm{s}$ integrated transversal filter as a FFE to improve signal integrity in serial electrical links. The transversal filter is implemented with a novel topology for improved testability, such that the FFE per-
nover novel topology for improved testability, such that the FFE per-
formance can be individually characterized, in both frequencyformance can be individually characterized, in both frequency-
and time-domain, which also helps to perform the coefficients and time-domain, which also helps to perform the coefficients
optimization of the 5-tap FFE. Apart from NRZ, duobinary signaling is implemented as an alternative modulation scheme, because of a higher spectral efficiency compared to NRZ, leading to a larger eye opening.

\section{REFERENCES}

[1] E. Song, J. Cho, W. Lee, M. Shin, and J. Kim, "A wide-band passive equalizer design on PCB based on near-end crosstalk and reflections for

Lett., vol. 18, no. 12, pp. 794-796, 2008 .
[2] J. H. Sinsky, M. Duelk, and A. Adamiecki, "High-speed electrical backplane transmission using duobinary signaling," IEEE Trans. Microw. Theory Tech., vol. 53, no. 1, pp. 152-160, 2005. Bauwelinck, "Experimental evaluation of NRZ and duobinary up to
$48 \mathrm{Gbit} / \mathrm{s}$ for clectrical backplanes," Electron. Lett., vol. 51 , no. 8, pp.

617-619, 2015.
[4] M.-S. Chen and C.-K. K. Yang, "A $50-64 \mathrm{~Gb} / \mathrm{s}$ serializing transmitter with a 4-tap, LC-ladder-filter-based FFE

[5] A. Hazneci and S. Voinigescu "A 49-Gb/s, 7-tap transversal filter in A. 18 azneci and S. Voinigescu, "A 49 -Gb/s, 7-tap transversal filter in
$0.18 \mu \mathrm{m} \mathrm{SiGe}$ BiCMOS for backplane equalization," in Proc. IEEE Compound Semicond. Integr. Circuit Symp. (CSICS), Oct. 2004, pp. 6] $101-104$.

Jye Keulenaer, G. Torfs, Y. Ban, R. Pierco, R. Vaernewyck, A. " 84 Gbit/s SiGe BiCMOS, B. Kozicki, X. Yin, and J. Bauwelinck,

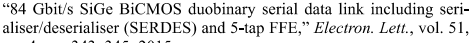
no. 4, pp. 343-345, 2015

[7] T. De Keulenaer, Y. Ban, G. Torfs, S. Sercu, J. De Geest, and J. Bauwelinck, "Measurements of millimeter wave test structures for Integrity (SPI), Ghent, Belgium, May 2014, pp. 1-4.

[8] J. Lee, M.-S. Chen, and H.-D. Wang, "Design and comparison of three 20-Gb/s backplane transceivers for duobinary, PAM 4, and NRZ data, A. Awny, L. Moeller, J. Junio, J. C. Scheytt, and A. Thiede, "De

A. Awny, L. Moel techniques for an $80 \mathrm{~Gb} / \mathrm{s} 1$ 1-tap decision feedback
and measurement techen and measurement techniques for an $80 \mathrm{~Gb} / \mathrm{s} 1$-tap decision feedback
equalizer," IEEE J. Solid-State Circuits, vol. 49, no. 2, pp. 452-470,
2014 . 